Supporting Information

\title{
In-Paper Self-Assembly of Cellulose Oligomers for the Preparation of All-Cellulose Functional Paper
}

\author{
Misaki Hanamura, ${ }^{\dagger}$ Toshiki Sawada,${ }^{\dagger, \ddagger}$ and Takeshi Serizawa ${ }^{*}, \dagger$ \\ $\dagger$ Department of Chemical Science and Engineering, School of Materials and Chemical \\ Technology, Tokyo Institute of Technology, 2-12-1-H121 Ookayama, Meguro-ku, Tokyo 152- \\ 8550 , Japan \\ $\$$ Precursory Research for Embryonic Science and Technology, Japan Science and Technology \\ Agency, 4-1-8 Honcho, Kawaguchi-shi, Saitama 332-0012, Japan \\ *E-mail: serizawa@mac.titech.ac.jp
}

\section{Contents:}

Number of pages: 8

Number of figures: 11 


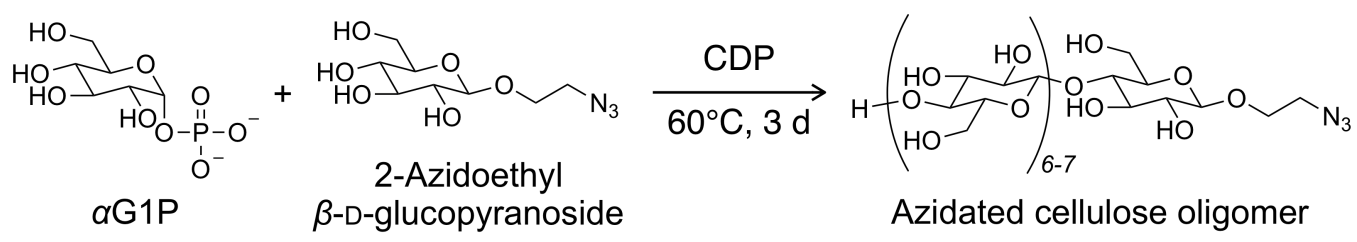

Figure S1. Synthesis scheme of azidated cellulose oligomers via cellodextrin phosphorylase (CDP)-catalyzed oligomerization of $\alpha$-D-glucose 1-phosphate $(\alpha \mathrm{G} 1 \mathrm{P})$ monomers from 2azidoethyl $\beta$-D-glucopyranoside primers.

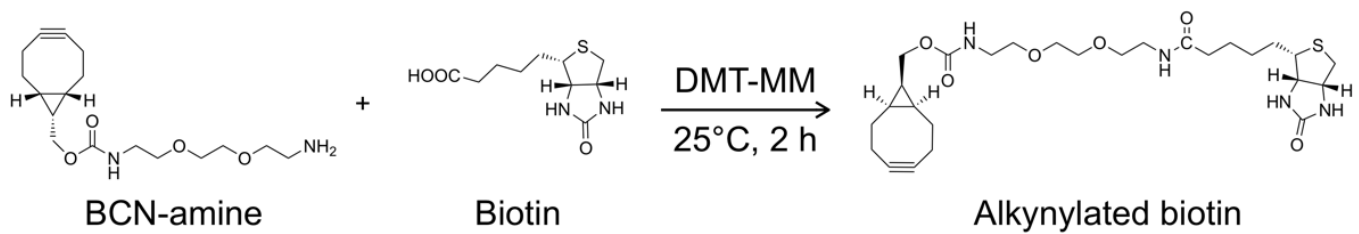

Figure S2. Scheme for the condensation reaction of BCN-amine and biotin mediated by a condensation reagent, 4-(4,6-dimethoxy-1,3,5-triazin-2-yl)-4-methylmorpholinium (DMT$\mathrm{MM})$.

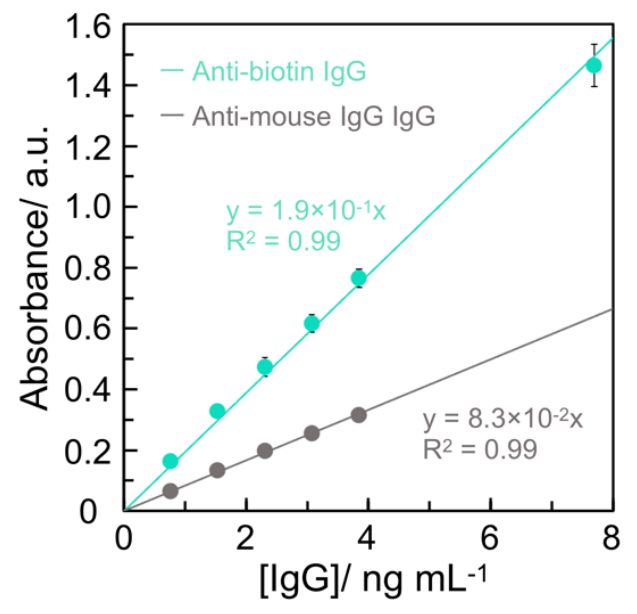

Figure S3. Dependences of the absorbance at $490 \mathrm{~nm}$ on the immunoglobulin $\mathrm{G}$ (IgG) concentrations. 
(a) (b)
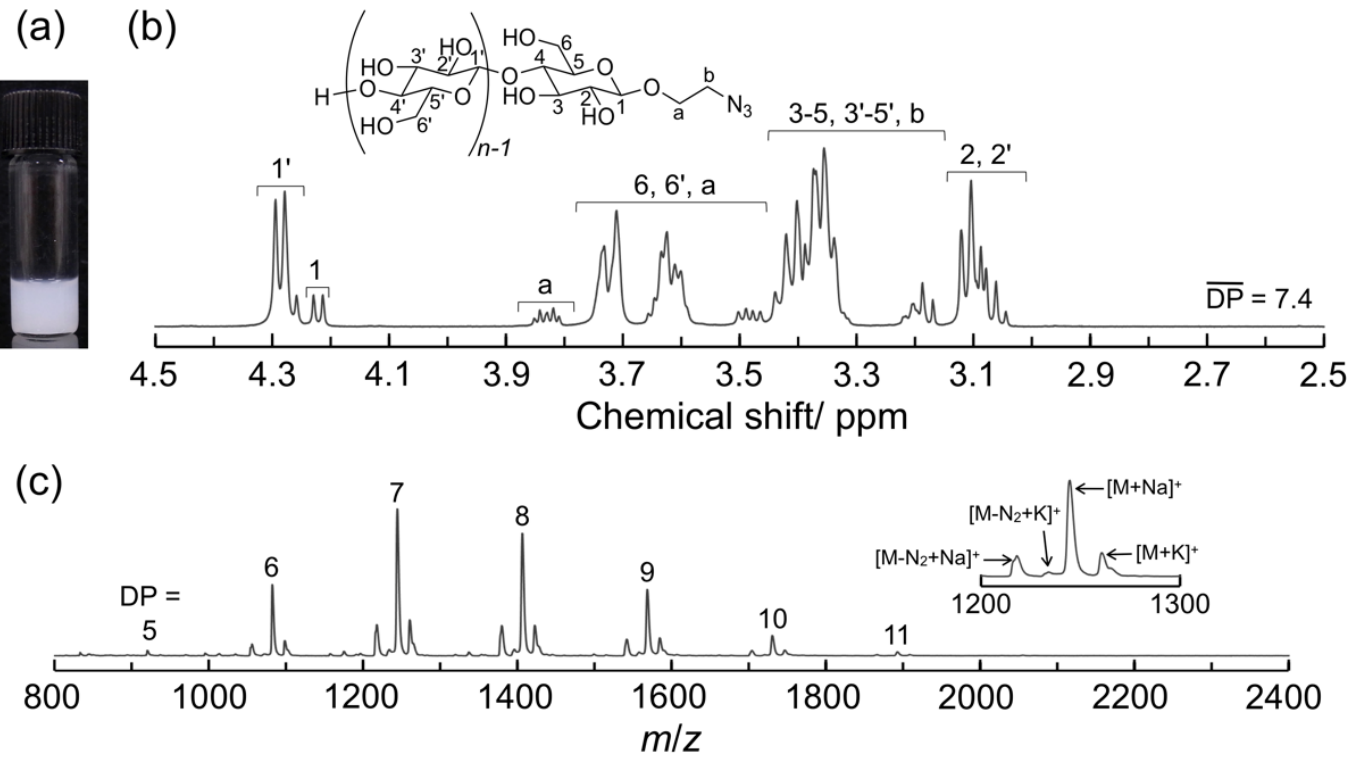

(d)

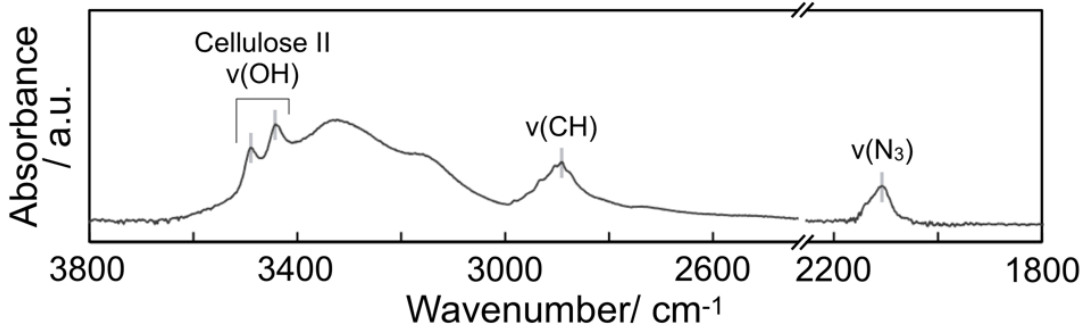

Figure S4. Characterizations of the synthesized azidated cellulose oligomers. (a) Photograph of the reaction solution. (b) ${ }^{1} \mathrm{H}$ nuclear magnetic resonance (NMR), (c) matrix-assisted laser desorption/ionization time-of-flight (MALDI-TOF) mass, and (d) Attenuated total reflectionFourier transform infrared (ATR-FTIR) absorption spectra of the oligomers. As shown in the inset of (c), the azido group lost $\mathrm{N}_{2}$ by fragmentation to become the amino group, similar to a previous report. ${ }^{1,2}$ However, it is noted that the ${ }^{1} \mathrm{H}$ NMR spectrum did not show a peak derived from cellulose oligomers with an amino group $(\delta=2.6),{ }^{3}$ suggesting the successful synthesis of azidated cellulose oligomers. 
(a)
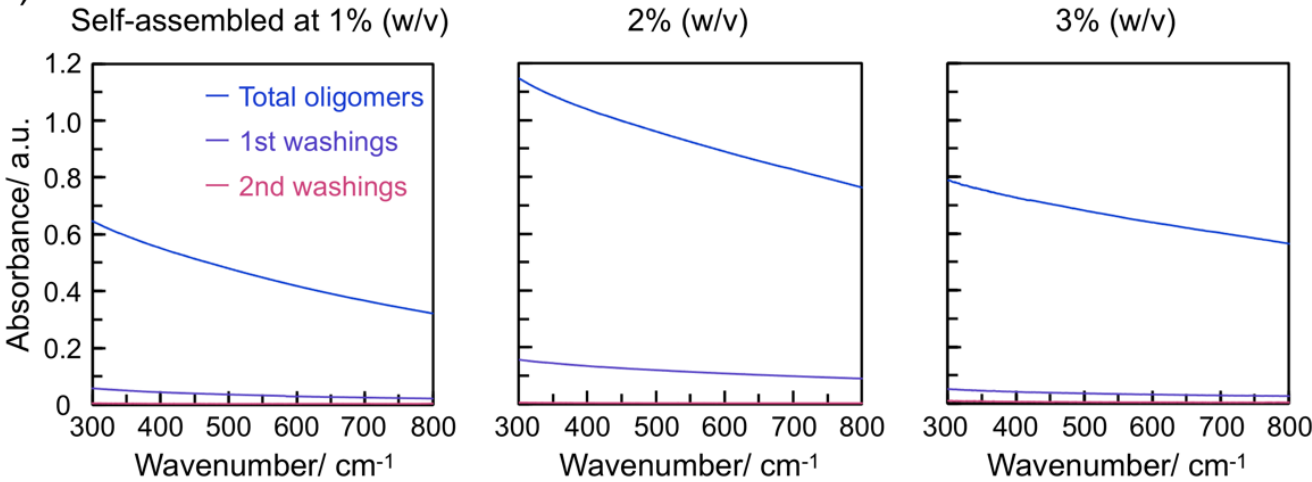

(b)

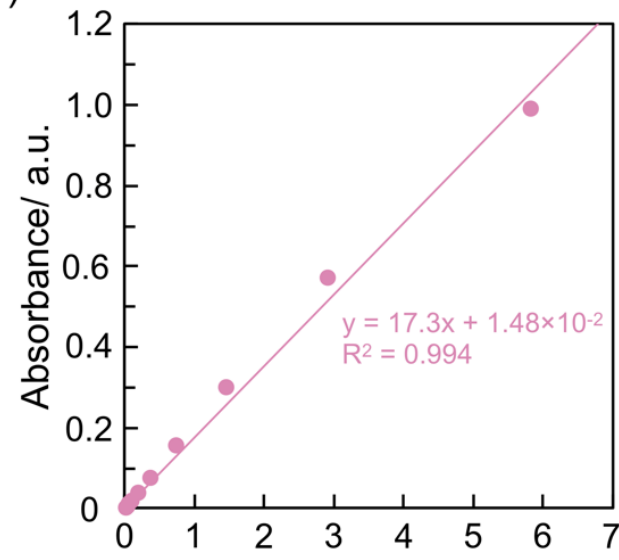

[Azidated cellulose oligomer]

/ $10^{-2} \%(\mathrm{w} / \mathrm{v})$

(c)
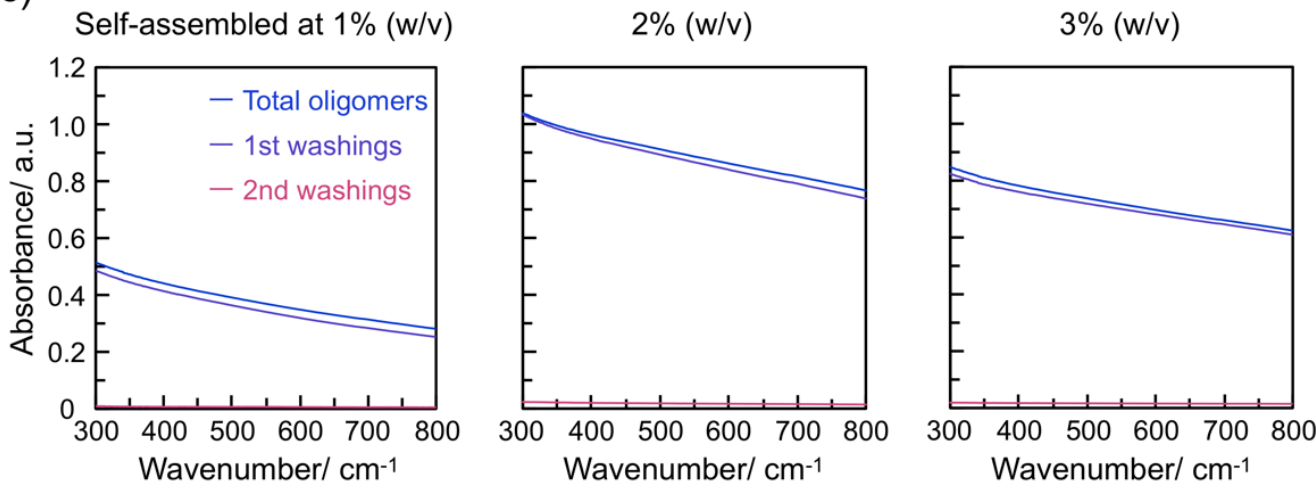

Figure S5. (a) Ultraviolet-visible (UV-Vis) absorption spectra of the washings after the inpaper self-assembly of azidated cellulose oligomers and the aqueous dispersion of the oligomers self-assembled in bulk conditions. (b) Absorbance at $500 \mathrm{~nm}$ against the concentration of azidated cellulose oligomers self-assembled in bulk conditions. The profile was used as a calibration curve. (c) UV-Vis absorption spectra of the washings after the impregnation of paper with azidated cellulose oligomers self-assembled in bulk solutions and the aqueous dispersion of the oligomers self-assembled in bulk conditions. When the oligomers self-assembled at $3 \%(\mathrm{w} / \mathrm{v})$, the spectra were measured after being diluted twice. 
(a)

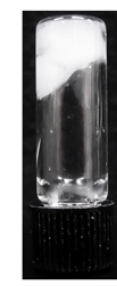

1

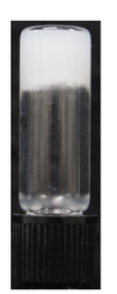

2 (b)

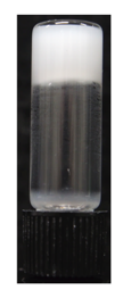

3

[Azidated cellulose oligomer] / \% (w/v) (c)
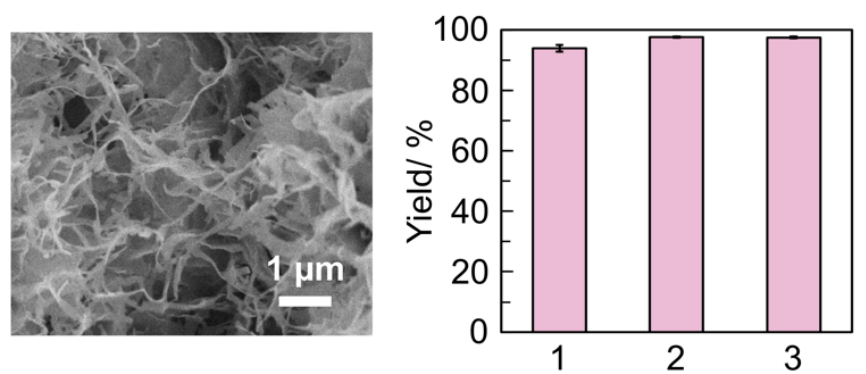

[Azidated cellulose oligomer] / \% (w/v)

(d)

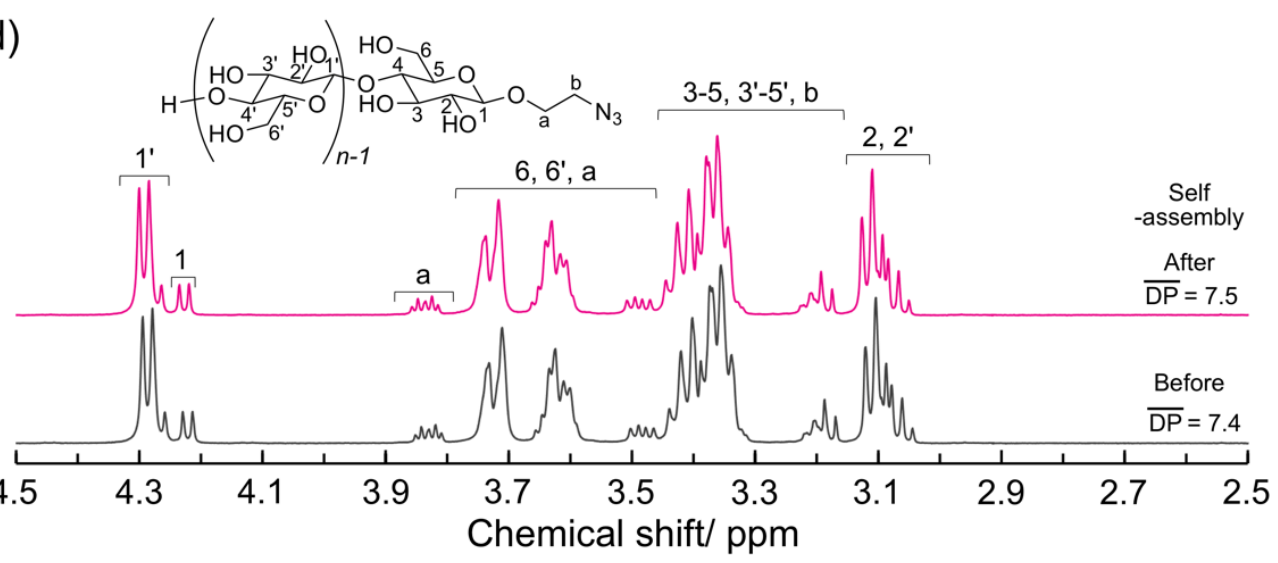

(e)

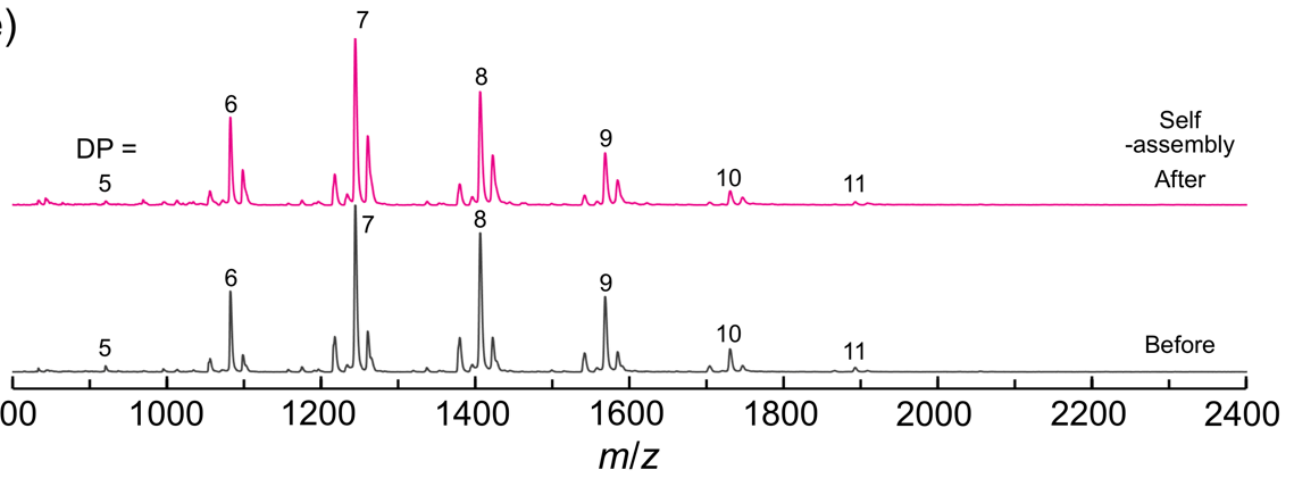

(f)

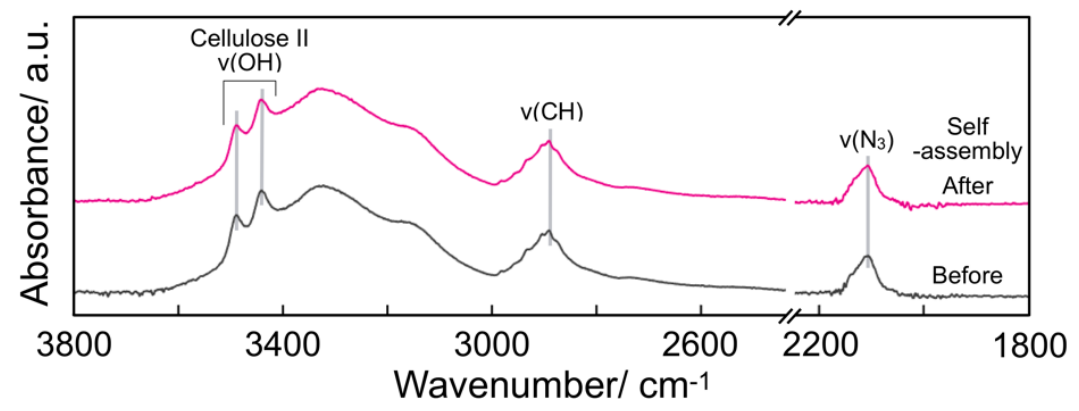

Figure S6. Characterizations of the self-assembled azidated cellulose oligomers. (a) Photographs of the self-assembled solutions at different concentrations. (b) Scanning electron microscopy (SEM) image of the self-assembled oligomers at 3\% (w/v). (c) Yields of the selfassembled oligomers. (d) ${ }^{1} \mathrm{H}$ NMR, (e) MALDI-TOF mass, and (f) ATR-FTIR absorption spectra of the oligomers before and after self-assembly. 
(a)

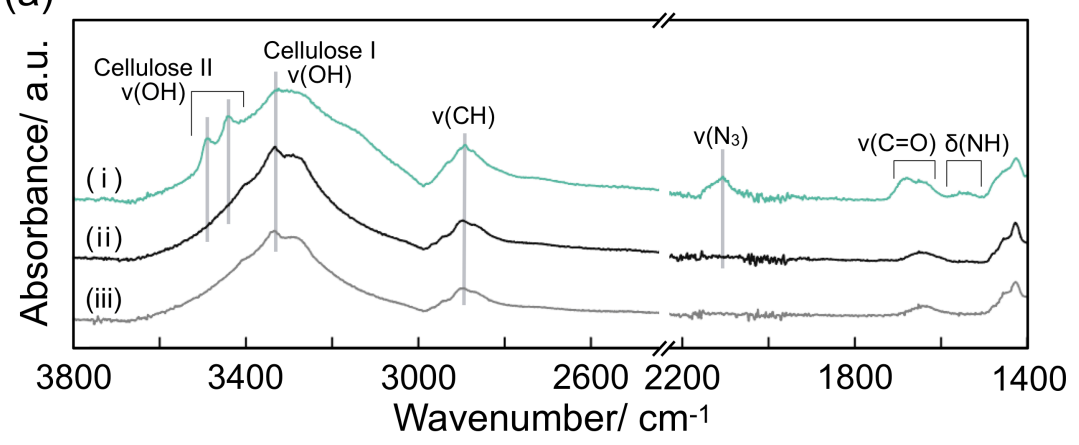

(b)

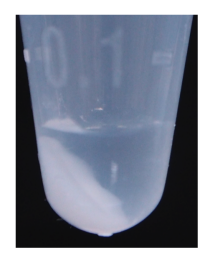

Figure S7. (a) ATR-FTIR absorption spectra of the biotinylated paper (i) before and (ii) after extraction of the biotinylated or non-biotinylated cellulose oligomers using aqueous alkaline solutions, and (iii) the pristine paper. In this experiment, the azidated paper after loading at 3\% $(\mathrm{w} / \mathrm{v})$ was used for biotinylation. (b) The precipitation of the extracted cellulose oligomers after neutralization-induced self-assembly.

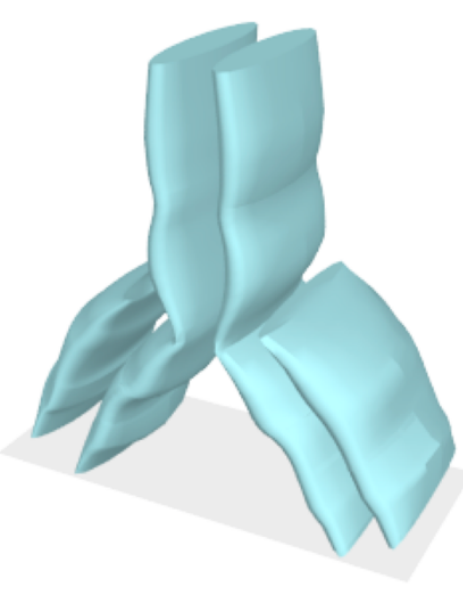

Cross-section area of IgG

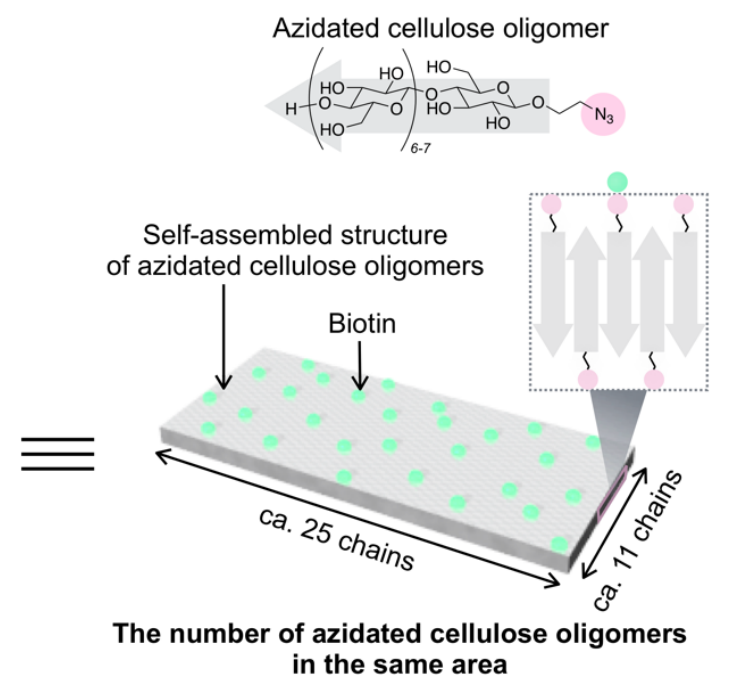

Figure S8. Comparative illustration of the cross-sectional area of IgG and the number of azidated cellulose oligomers with the cellulose II allomorph in the same area. Human fulllength IgG4 was used as a model size of IgG (PDB code: 5DK3). The right image schematically shows $20 \%$ biotinylation. 


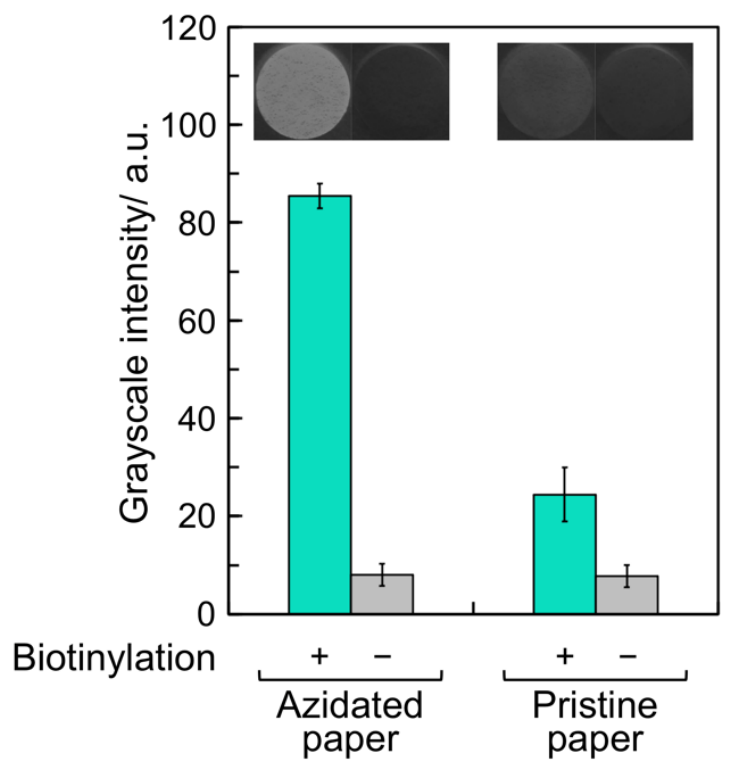

Figure S9. Grayscale intensities for photographs of the azidated and pristine papers with $(+)$ and without $(-)$ being subjected to the biotinylation procedure after the enzymatic reaction for IgG detection. The insets show the grayscale images for each paper.

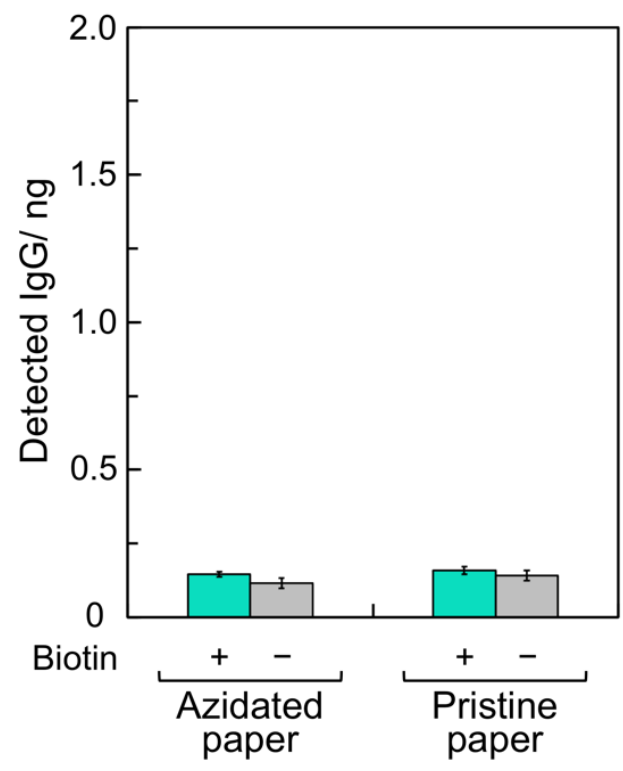

Figure S10. Quantities of detected anti-biotin IgG using the azidated and pristine papers with $(+)$ and without $(-)$ being subjected to physical adsorption of intact biotin without the alkynylation procedure after the enzymatic reaction for IgG detection. 


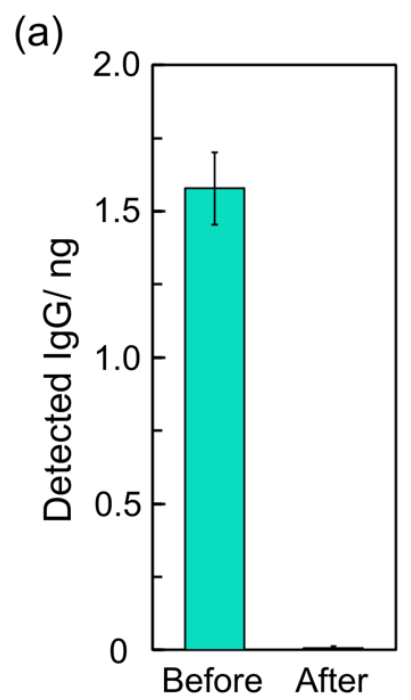

(b)

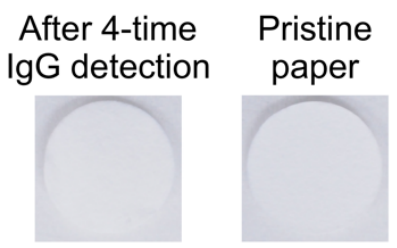

Figure S11. (a) Quantities of detected IgG using the biotinylated paper before and after treatment with acidic buffer solutions via enzymatic reactions. (b) Photographs of the biotinylated paper after IgG detection four times and the washing procedure.

\section{References}

(1) Raynaud, J.; Absalon, C.; Gnanou, Y.; Taton, D. N-Heterocyclic Carbene-Induced Zwitterionic Ring-Opening Polymerization of Ethylene Oxide and Direct Synthesis of $\alpha, \omega$ Difunctionalized Poly(ethylene oxide)s and Poly(ethylene oxide)- $b$-poly( $\varepsilon$-caprolactone) Block Copolymers. J. Am. Chem. Soc. 2009, 131, 3201-3209.

(2) Guillaneuf, Y.; Dufils, P. E.; Autissier, L.; Rollet, M.; Gigmes, D.; Bertin, D. Radical Chain End Chemical Transformation of SG1-Based Polystyrenes. Macromolecules 2010, 43, 91100 .

(3) Nohara, T.; Sawada, T.; Tanaka, H.; Serizawa, T. Enzymatic Synthesis and Protein Adsorption Properties of Crystalline Nanoribbons Composed of Cellulose Oligomer Derivatives with Primary Amino Groups. J. Biomater. Sci. Polym. Ed. 2017, 28, 925-938. 